\title{
Electron anisotropic scattering in gases: A formula for Monte Carlo simulations
}

\author{
A. Okhrimovskyy, A. Bogaerts, and R. Gijbels \\ Department of Chemistry, University of Antwerp, Universiteitsplein 1, B-2610 Wilrijk-Antwerp, Belgium \\ (Received 22 June 2001; revised manuscript received 26 November 2001; published 27 February 2002)
}

\begin{abstract}
The purpose of this Brief Report is to point out the mistake in a formula for anisotropic electron scattering, previously published in Phys. Rev. A 41, 1112 (1990), which is widely used in Monte Carlo models of gas discharges. Anisotropic electron scattering is investigated based on the screened Coulomb potential between electrons and neutral atoms. The approach is also applied for electron scattering by nonpolar neutral molecules. Differential cross sections for electron scattering by $\mathrm{Ar}, \mathrm{N}_{2}$, and $\mathrm{CH}_{4}$ are constructed on the basis of momentum and integrated cross sections. The formula derived in this paper is useful for Monte Carlo simulations of gas discharges.
\end{abstract}

DOI: 10.1103/PhysRevE.65.037402

PACS number(s): 52.20.Fs, 34.80.Bm, 31.15.Qg

\section{INTRODUCTION}

In a Monte Carlo model, the trajectory of the particles (e.g., electrons) is followed by Newton's laws, whereas the collisions are treated by random numbers (i.e., for the time between two collisions, the kind of collision, the new energy, and direction after scattering).

To describe electron scattering in gases, a formula for anisotropic scattering needs to be used. In many papers (e.g., $[1-5])$, the expression for the normalized differential scattering cross section $I(\epsilon, \chi)$ introduced by Surendra and coworkers $[6,7]$ is used:

$$
I(\epsilon, \chi)=\frac{\epsilon}{4 \pi\left[1+\epsilon \sin ^{2}(\chi / 2)\right] \ln (1+\epsilon)},
$$

which is based on look-alike, screened-Coulomb scattering. $\epsilon$ is defined as the energy of electrons before collision (in eV). However, it appears that this formula is incorrect and misses some scientific background (e.g., the energy has to be reduced to a dimensionless form by introducing some universal energy value).

Some investigations of anisotropic scattering of electrons have already been made in particular cases [8-11]. In the present paper, we will derive a different analytical expression for anisotropic scattering of electrons, which is generally valid in atomic and nonpolar molecular gases. The general formula will then be applied to a number of atomic and nonpolar molecular gases, to derive formulas that are easy to implement in Monte Carlo algorithms.

\section{DIFFERENTIAL CROSS SECTION FOR LOOK-ALIKE SCREENED-COULOMB SCATTERING}

In an atomic gas with atomic number $Z$ the electronneutral interaction potential can be approximated by the screened Coulomb potential

$$
U(r)=Z E_{0} \frac{r_{0}}{r} \exp \left(-\frac{r}{r_{0}}\right) .
$$

Here $E_{0}$ is the atomic unit of energy $\left(E_{0}=27.21 \mathrm{eV}\right)$ and $r_{0}$ is the Bohr radius $\left(r_{0}=0.529 \times 10^{-10} \mathrm{~m}\right)$. In this expression we used as a screening radius the "outer boundary" of the atom, which is about one Bohr radius [12] according to the Thomas-Fermi model of an atom.

In the first Born approximation of the quantum mechanics theory of scattering $[12,13]$ the normalized differential cross section for screened-Coulomb scattering of an electron is

$$
I(\varepsilon, \chi)=\frac{1}{4 \pi} \frac{1+8 \varepsilon}{(1+4 \varepsilon-4 \varepsilon \cos \chi)^{2}},
$$

where $\varepsilon=E / E_{0}$ is the dimensionless energy and $E$ is the relative energy of electrons.

The probability that an electron with dimensionless en$\operatorname{ergy} \varepsilon$ is scattered with an angle $\chi$ can be found by integrating Eq. (3) over all possible scattering angles,

$$
P(\varepsilon, \chi)=\frac{(1+8 \varepsilon) \sin ^{2} \frac{\chi}{2}}{1+8 \varepsilon \sin ^{2} \frac{\chi}{2}}
$$

Finally, to find the scattering angle, a random number $R$ uniformly distributed in the interval $[0,1]$ is compared with the probability of scattering; and the reverse function should be found,

$$
\cos \chi=1-\frac{2 R}{1+8 \varepsilon(1-R)} .
$$

Equation (5) is very convenient for Monte Carlo modeling purposes of the electron behavior in atomic gases. We will now derive a similar expression for electron scattering with nonpolar molecules $\left(\mathrm{CH}_{4}, \mathrm{~N}_{2}, \mathrm{O}_{2}\right.$, etc.), assuming the same functional form of differential cross section as for the idealized atom. Equation (3) can be rewritten in the more general form using one fitting parameter [9], 

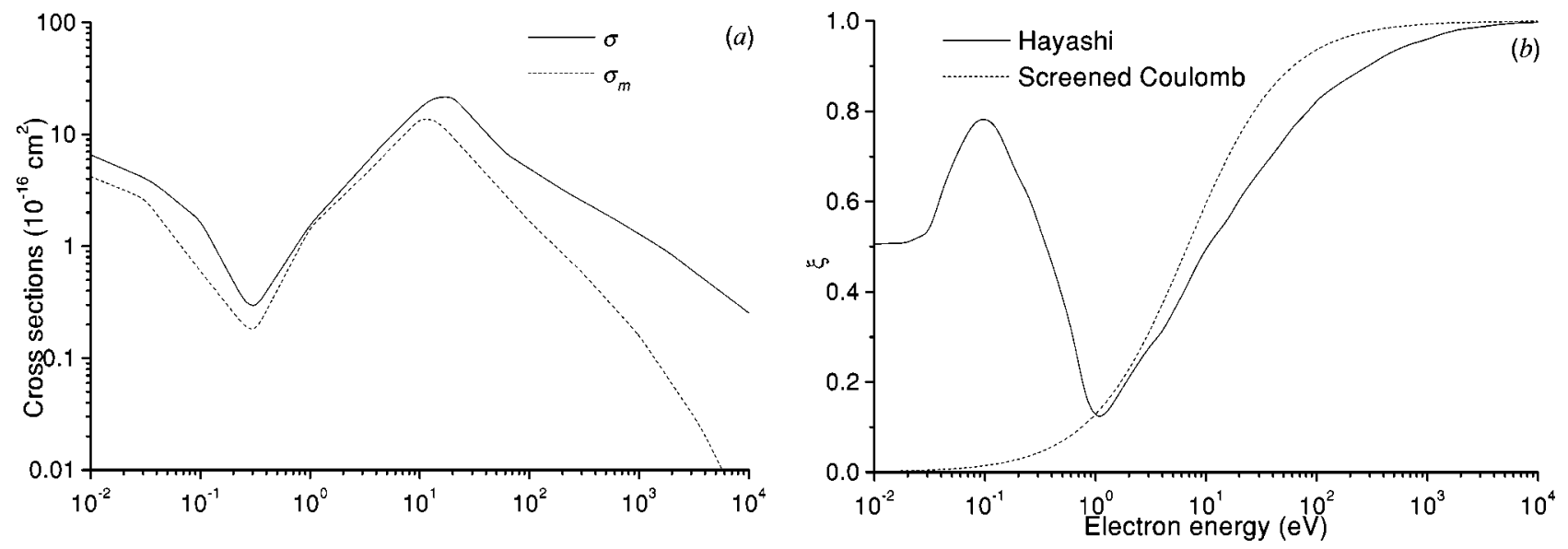

FIG. 1. (a) Integrated elastic $\sigma$ (solid) and momentum transfer $\sigma_{m}$ (dashed) cross sections for Ar from Hayashi [17]; (b) derived $\xi$ based on the data of Hayashi (solid) and for the screened-Coulomb potential (dashed) as a function of electron energy.

$$
I(\varepsilon, \chi)=\frac{1}{4 \pi} \frac{1-\xi^{2}(\varepsilon)}{[1-\xi(\varepsilon) \cos \chi]^{2}}
$$

similar to the procedure described by Phelps [14]. It should be mentioned that we used the notation $\xi(\varepsilon)$, which is equivalent to " $1-2 * \beta(n)$ " in Phelps notation [14]. This expression reduces to conventional screened-Coulomb scattering if $\xi=4 \varepsilon /(1+4 \varepsilon)$. In general, $\xi$ is a function of energy (see below), which varies in the interval $(-1,1)$. Depending on the kind of interaction potential, $\xi$ is a different function of energy. Hence, this yields a different scattering formula.

Equally, the probability of electron scattering with an angle $\chi$ can be calculated. And again, the scattering angle can be found by comparing a random number $R$ uniformly distributed in the interval $[0,1]$ with the probability of scattering; and reversing the function,

$$
\cos \chi=1-\frac{2 R(1-\xi)}{1+\xi(1-2 R)}
$$

This expression is generally valid to describe the scattering of electrons by nonpolar molecules, and it is convenient to use in electron Monte Carlo models when the function $\xi(\varepsilon)$ is known.

In the case of conventional screened-Coulomb interaction, $\xi=4 \varepsilon /(1+4 \varepsilon)$ (see above) and it can vary between 0 and 1 . In the low energy limit, $\xi \rightarrow 0$ and the scattering becomes isotropic [i.e., $I(\varepsilon, \chi)=$ constant]. When the energy of the scattered electron goes to infinity, $\xi \rightarrow 1$ and the scattering corresponds to conventional Coulomb scattering and becomes in the forward direction.

In general, the function $\xi(\varepsilon)$ can be deduced from the ratio of integrated and momentum transfer cross sections, based on the procedure described by Phelps [14]. Indeed, the ratio of momentum transfer and integrated cross section can be obtained from the normalized differential cross section by multiplying with factor $2 \pi(1-\cos \chi)$ and integrating over all angles. For $I(\varepsilon, \chi)$ from Eq. (6) it yields for this ratio

$$
\frac{\sigma_{m}}{\sigma}=\frac{1-\xi}{2 \xi^{2}}\left((1+\xi) \ln \frac{1+\xi}{1-\xi}-2 \xi\right)
$$

This expression is generally valid for all kinds of interactions between electrons and atoms or nonpolar molecules. In the literature, some experimental data are available for both integrated and momentum transfer cross sections as a function of electron energy, for electron elastic collisions with atoms or molecules $[8,15-18]$. Hence, the function $\xi(\varepsilon)$ for a specific interaction can be obtained from the ratio of experimental cross sections $\sigma_{m}(\varepsilon) / \sigma(\varepsilon)$. Finally, when $\xi(\varepsilon)$ is known, the formula for the scattering angle can be obtained with Eq. (7).

The main idea of the procedure to construct differential cross sections based on the screened-Coulomb functional dependence from the data of the integrated and momentum transfer cross sections was first published by Belenguer and Pitchford [9]. In the case of the resonance atom-atom scattering Phelps et al. [19] proposed a three fitting parameter procedure. Porter et al. [11] proposed even a four fitting parameter approximation for the differential cross section of the electron scattering in $\mathrm{N}_{2}$. But with all these manyparameter fitting expressions it is difficult to find the scattering angle that is needed for Monte Carlo modeling. Since it is the purpose of our paper to derive such formula for the scattering angle, we present here a single parameter fitting expression.

The differential cross section constructed in this way for electron scattering in $\mathrm{Ar}$ is in good agreement with experimental data of Vuskovik and Kurepa [20]. It is clear that with this approach, the main features of scattering are captured, i.e., the scattering is approximately isotropic at low energies and becomes peaked in the forward direction at high energy.

This method is generally valid for all atoms or nonpolar molecules. Polar molecules, on the other hand, are characterized by a dipole moment, and therefore a screened-Coulomblike interaction potential is not valid. Therefore, the procedure is not applicable to describe electron collisions with 

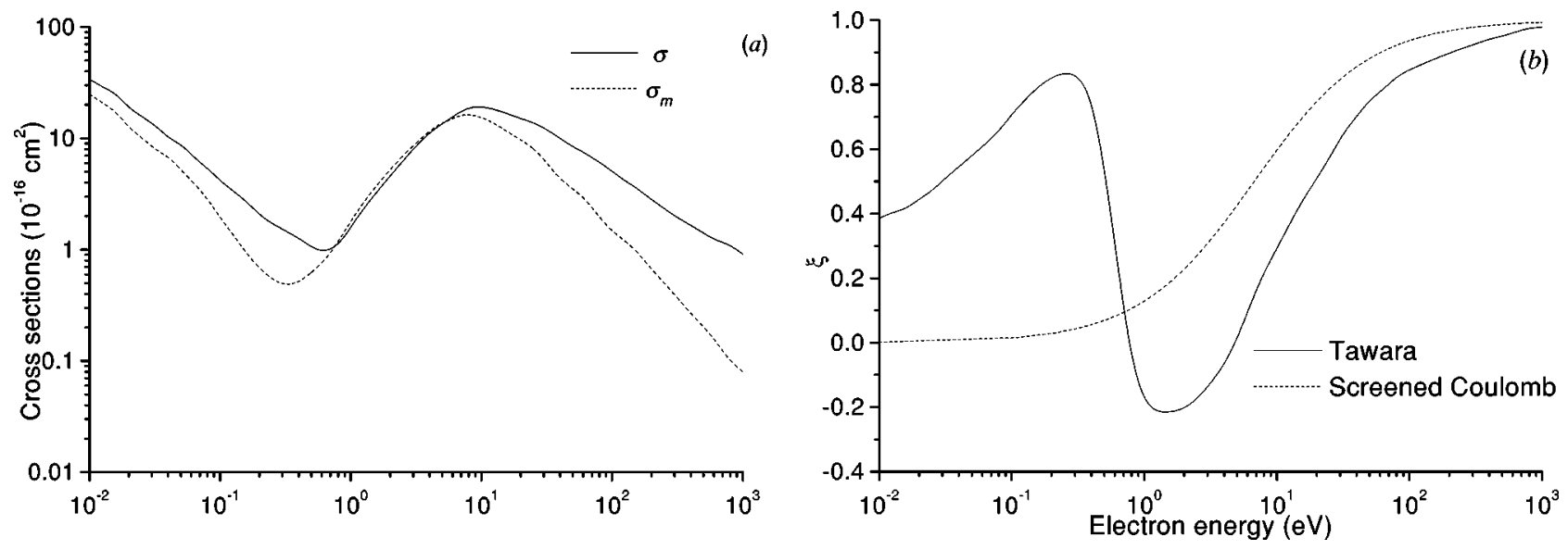

FIG. 2. (a) Integrated elastic $\sigma$ (solid) and momentum transfer $\sigma_{m}$ (dashed) cross sections for $\mathrm{CH}_{4}$ from Tawara et al. [18]; (b) derived $\xi$ based on the data of Tawara (solid) and for the screened-Coulomb potential (dashed) as a function of electron energy.

polar molecules. However, it can be a reasonable approximation for anisotropic scattering if no other data are available.

\section{THE SCATTERING FORMULA ILLUSTRATED FOR A FEW NONPOLAR GASES}

To illustrate the proposed approach, we have calculated the parameter $\xi$ as a function of energy for a few nonpolar gases, i.e., for argon (as atomic gas), methane (as a polyatomic molecule with spherical symmetry), and nitrogen (as a linear molecule).

For $\mathrm{Ar}$ we used the integrated and momentum transfer elastic cross section data from Hayashi [17]. These cross sections are presented in Fig. 1(a). The derived energy dependence of $\xi$ for electron anisotropic scattering with Ar atoms, is presented in Fig. 1(b) (solid line).

For $\mathrm{CH}_{4}$ we used the integrated and momentum transfer elastic cross section data from Tawara et al. [18]. The cross sections as a function of energy are presented in Fig. 2(a) and the corresponding $\xi$ as a function of electron energy is illustrated in Fig. 2(b) (solid line).

Finally, for $\mathrm{N}_{2}$ we used the integrated and momentum

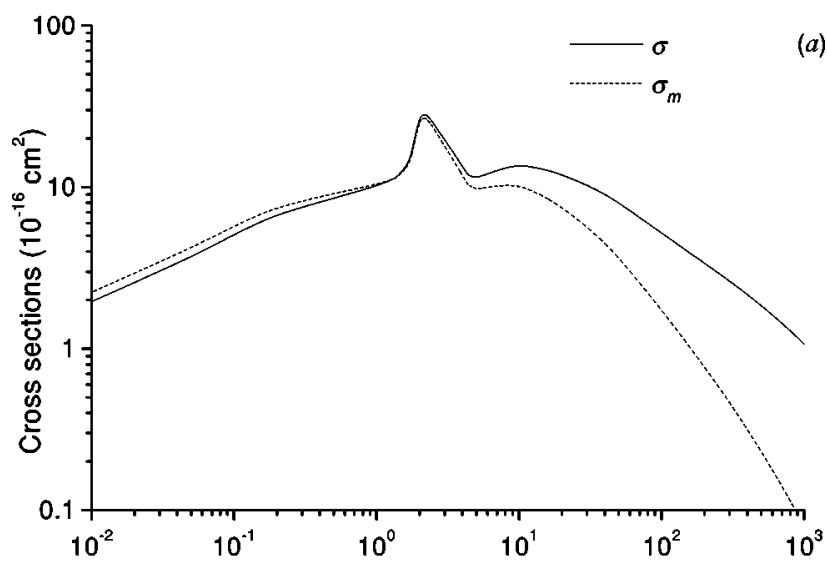

transfer elastic cross section data from Phelps and Pitchford [8]. The cross sections are plotted as a function of $E$ in Fig. 3 (a). For electron scattering on $\mathrm{N}_{2}$, we were able to make an empirical fit of $\xi(E)$,

$$
\xi(E)=\frac{0.065 E+0.26 \sqrt{E}}{1+0.05 E+0.2 \sqrt{E}}-\frac{12 \sqrt{E}}{1+40 \sqrt{E}}
$$

where $E$ symbolizes the electron energy in $\mathrm{eV} . \xi(E)$ is given in Fig. 3(b).

In all these figures the corresponding values for $\xi(\varepsilon)$ are also presented for the conventional screened-Coulomb potential (dashed lines). It is clear that for an atomic gas such as Ar, the approximation of the conventional screenedCoulomb potential is more or less applicable for energies higher than $1 \mathrm{eV}$. In such case the expression (5) can be used to find out the scattering angle. At lower energy the Ramsauer effect becomes important and the screened-Coulomb approach is not valid anymore. For molecular gases the approximation of the screened-Coulomb potential is clearly not

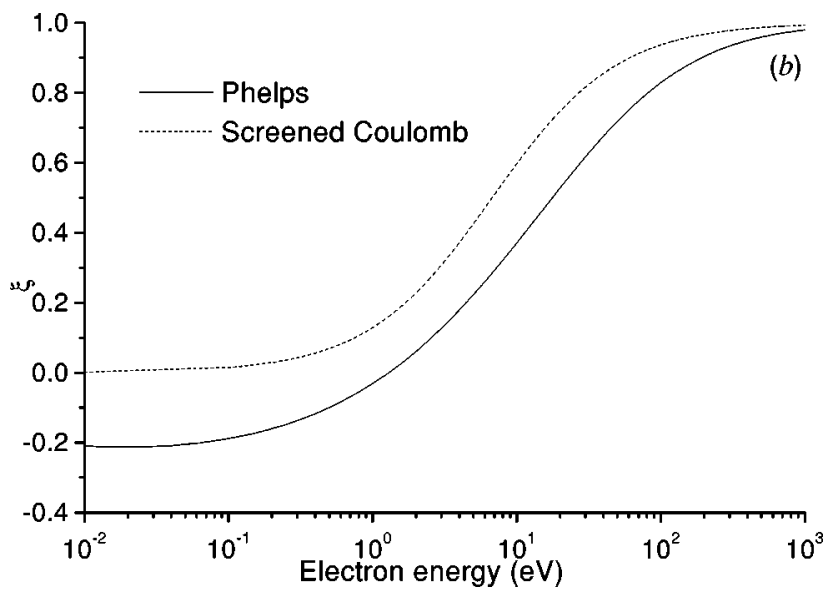

FIG. 3. (a) Integrated elastic $\sigma$ (solid) and momentum transfer $\sigma_{m}$ (dashed) cross sections for $\mathrm{N}_{2}$ from Phelps and Pitchford [8]; (b) derived $\xi$ based on the data of Phelps and Pitchford (solid) and for the screened Coulomb potential (dashed) as a function of electron energy. 
valid [see Figs. 2(b) and 3(b)] and the more general formula (6) should be used.

\section{CONCLUSION}

A simple expression for the scattering angle useful for Monte Carlo modeling has been deduced for electron collisions with atomic gases. This expression has been extended to a one fitting parameter formula that can be used to find the scattering angle for electron collisions with nonpolar mo- lecular gases. For elastic collisions of electrons with $\mathrm{Ar}, \mathrm{N}_{2}$, and $\mathrm{CH}_{4}$ the energy dependence of this single parameter is presented in a broad energy range.

\section{ACKNOWLEDGMENTS}

A.O. and A.B. acknowledge financial support from the Flemish Fund for Scientific Research (FWO). The authors also thank A. Phelps, N. Aleksandrov, and M. Surendra for the helpful discussions on the subject of this paper.
[1] A. Bogaerts, M. Van Straaten, and R. Gijbels, Spectrochim. Acta, Part B 50, 179 (1995).

[2] S. Kondo and K. Nanbu, J. Phys. D 32, 1142 (1999).

[3] E. Shidoji, H. Otake, N. Nakano, and T. Makabe, Jpn. J. Appl. Phys., Part 1 38, 2131 (1999).

[4] H. Rau, J. Phys. D 33, 3214 (2000).

[5] P. Hartmann, Z. Donko, G. Bano, L. Szalai, and K. Rozsa, Plasma Sources Sci. Technol. 2, 183 (2000).

[6] M. Surendra, D. B. Graves, and G. M. Jellum, Phys. Rev. A 41, 1112 (1990).

[7] V. Vahedi and M. Surendra, Comput. Phys. Commun. 87, 179 (1995).

[8] A. V. Phelps and L. C. Pitchford, Phys. Rev. A 31, 2932 (1985).

[9] Ph. Belenguer and L. C. Pitchford, J. Appl. Phys. 86, 4780 (1999).

[10] S. Longo and M. Capitelli, Plasma Chem. Plasma Process. 14, 1 (1994).

[11] H. S. Porter, F. Varosi, and H. G. Mayr, J. Geophys. Res.,
[Space Phys.] 92, 5933 (1987).

[12] L. D. Landau and E. M. Lifshiz, Quantum Mechanics: Nonrelativistic Theory (Pergamon, Oxford, 1977).

[13] L. I. Schiff, Quantum Mechanics (McGraw-Hill, New York, 1949).

[14] A. V. Phelps, ftp://jila.colorodo.edu/collision_data/electron.txt (A. Phelps asked the authors to point out that there was a mistake in this file about fitting of the parameter of the differential cross section for $\mathrm{N}_{2}$. In the meantime it is corrected.)

[15] F. J. Heer and R. H. Jansen, J. Phys. B 10, 3741 (1977).

[16] L. L. Alves and C. M. Ferreira, J. Phys. D 24, 581 (1991).

[17] M. Hayashi, IPPJ-AM Research Report No. 19, 1981 (unpublished).

[18] H. Tawara, Y. Itikawa, H. Nishimura, H. Takana, and Y. Nakamura, Research Report NIFS-data series No. 6, 1990 (unpublished).

[19] A. V. Phelps, C. H. Gene, and J. P. Bruke, J. Phys. B 33, 2965 (2000).

[20] L. Vuskovik and M. V. Kurepa, J. Phys. B 9, 837 (1976). 